\title{
Design and Implementation on Physical Fitness and Health Standards Query System for College Students
}

\author{
Zhu Li \\ College of Physical Education, Dalian University, Dalian, 116622, China \\ 48269162@qq.com
}

Keywords: college students; physical fitness and health standards; query system; design and implementation; requirement analysis; functional design; framework structure

\begin{abstract}
Schools must implement the national physical exercise standards, and through the evaluation to promote students to actively participate in physical exercise, improve physical health, promote the overall development of body, and become high-quality builders with right sports awareness and healthy lifestyle. In order to facilitate the inquiry from students and teachers, this paper is based on the idea and method of software engineering design, providing technical support for system development. The system is based on "National student's physical fitness and health standards" and SSH framework structure design and implementation. The main research includes requirement analysis, functional design, business layer implementation and persistence layer implementation. The research results of this paper solve the key technical problems in system development and have important guiding significance to improve the efficiency and quality of software development.
\end{abstract}

\section{Introduction}

"National student physical health standard" (hereinafter referred to as "standard"), is to implement health first guiding ideology, effectively strengthen school sports work, promote students to actively participate in physical exercise, develop good exercise habits, promote normal development and comprehensive development of the body, improve the physical health standard. "Standard", is one of the basic conditions for students' physical health evaluation standards and whether students can graduate, is an educational mean to inspire students actively participate in physical exercise, promote the healthy development of students' physique, guide the young students to have healthy personality. The "health first" guiding ideology implemented, give full play to the role of school sports in quality education [1,2]. "Standard" is used for students from full-time primary school, middle school, regular high school, secondary vocational school, ordinary colleges and universities.

Software framework is a series of related abstraction modes that guide the design of all aspects of large software system. Software framework completes the transition from service-oriented to technology-oriented, with the advantages of shortening development cycle, improving software quality, reducing software costs, making easy to modify and maintaining. J2EE is a java-based server-side programming technology, J2EE platform is a multi-level distributed application model, retains existing IT resources, with high development efficiency, support for heterogeneous environments, excellent scalability and stable availability. The logical function of the J2EE platform application is encapsulated into the component, the client layer component, the Web layer component, the business logic layer component and the business layer component, and the client implements the database interaction through the browser.

The development of college students' physical health standard query system plays an important role. On the one hand, students can test their physical situation at any time according to the standard, and the implementation of targeted projects training, achieve the role by improving performance and strengthening physical health; the other hand, teachers in the teaching and researching process, also can query at any time, avoid the drawbacks of reading paper materials. This paper is based on the 
SSH framework of J2EE platform, designed specifically for college students' physical health standard query, and provides technical support for system development.

\section{Requirement Analysis}

"Standard" will divided the tested students into two groups, first and second grade is divided into a group, third grade and fourth grade as a group. The test items are shown in Table 1.

Table 1. Test item of physical fitness and health

\begin{tabular}{ccc}
\hline No & Test item & Weight (\%) \\
\hline 1 & Body mass index (BMI) & 15 \\
2 & Vital capacity & 15 \\
3 & 50 meter dash & 20 \\
4 & Sitting trunk flexion & 10 \\
5 & Standing long jump & 10 \\
6 & Pull-up (SchoolBoy), 1 minute sit ups (SchoolGirl) & 10 \\
7 & 1000 meter run (SchoolBoy), 800 meter run (SchoolGirl) & 20 \\
\hline
\end{tabular}

Notes: BMI=body mass/ the square of height (The body weight is $\mathrm{kg}$, The unit of height is $\mathrm{m}$ )

From Table 1, "BMI" for the body shape indicators, "Vital capacity" for the body function indicators, the other for the physical quality indicators. The total score of the test is made up of the sum of the standard points and the additional points. The standard is divided by the sum of the individual indicators and the weight of the product, full mark 100 points. Additional points based on the measured results to determine, that is more than 100 points on the score points to add points, full mark 20 points; boys plus points indicators for the upward and 1000 meters run, girls add points for 1 minute sit-up and 800 meters run, the index points are all 10 points [3-5].

According to the total score of the test rating: 90.0 points and above for excellent, 80.0-89.9 for good, 60.0-79.9 points for the pass, 59.9 points and below failed. Before students test scores achieve good and above, they can participate in the assessment and appraisement; achieve excellent, before they can get sports credits. If the test result is failed, the test will be approved once in the current year, and if the test result will not be passed, the year point is failed. Achieve less than 50 points deal with by finishing or processing.

\section{Functional Design}

Functional design is used to describe with the hierarchical structure of software functions. A rectangular box in the structure chart represents a module. The lines between the rectangles represent the call. The rectangle on the upper layer represents the module and the module is called on the lower rectangle. Modular design should follow the principles of abstraction, gradual refinement, modularity and information concealment. Modular design of the software can have different levels of abstraction. At the highest level of abstraction, you can use the language description method of the environment in which the problem is located. And in the lower level of abstraction, the use of process methods; step by step refinement is a top-down design strategy, the software architecture, in accordance with the top-down approach to the various levels of process details and data The module is a part of the program can be logically separated, modular refers to the "software is a number of discrete parts of the dispersion degree, that is, the degree of software modularity"; information concealment, that is, the implementation details of each module for other modules is hidden.

According to the demand analysis, the system is decomposed into two subsystems. Among them, the query subsystem, for student users and teachers, for the query of physical health standards; maintenance subsystem, for system administrator users, for user management and system maintenance. Reference to the relevant literature [6,7], modular design results shown in Fig. 1. 


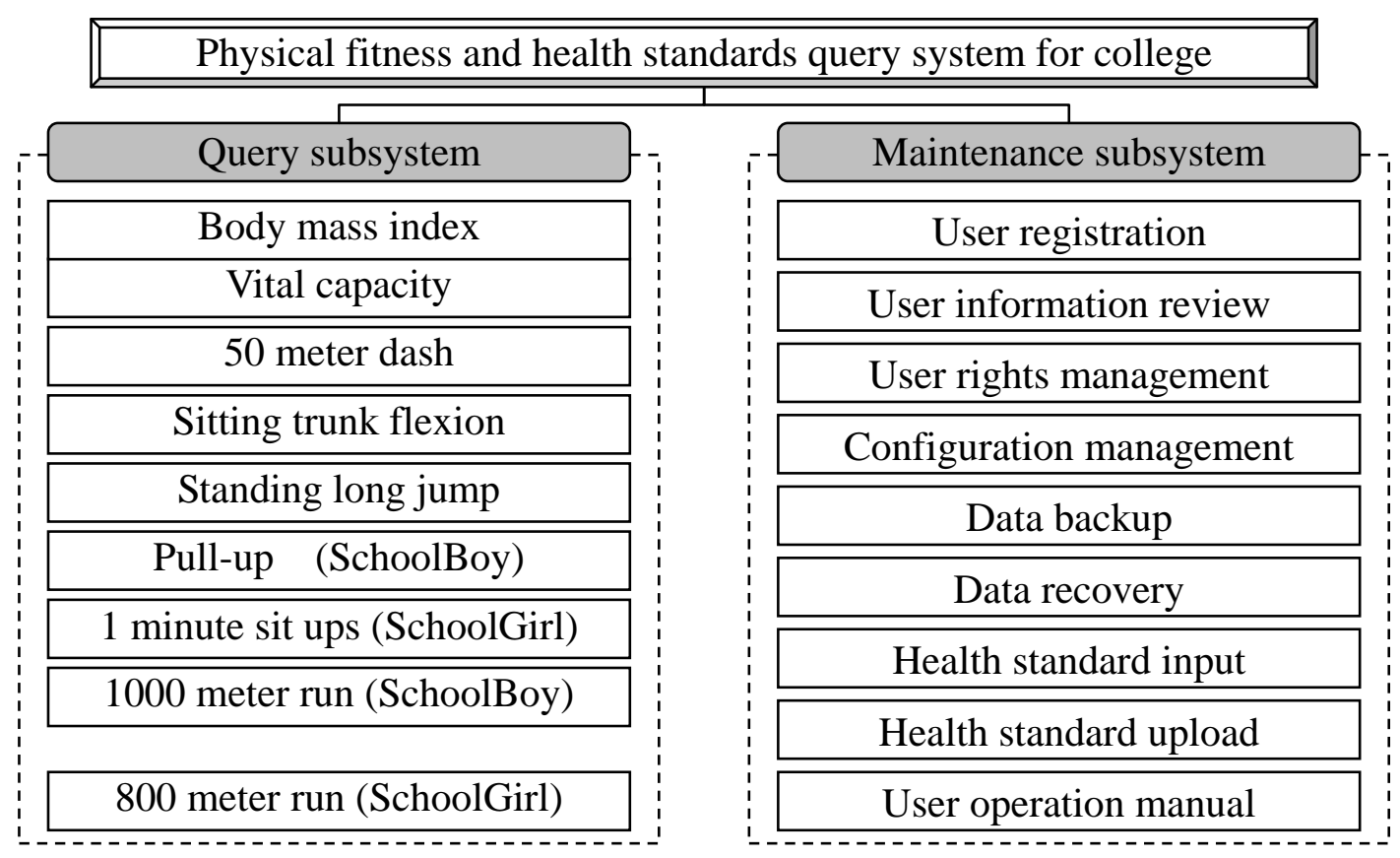

Fig. 1. Functional framework on Physical fitness and health standards query system for college students

\section{Business Layer Implementation}

Business layer is located between the control layer and the persistence layer. The business layer is used to deal with the business logic. The business logic is managed by the Spring framework to implement the business interface, by calling the DAO Interface to complete the operation with the persistence layer [8]. The business layer structure is shown in Fig. 2.

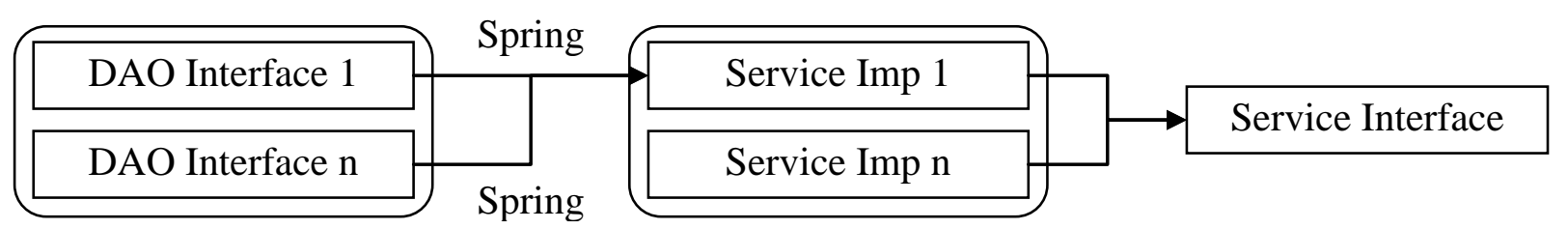

Fig. 2. Business layer structure

(1) Define business logic component interface. Interfaces are a set of declarations that improve the flexibility and scalability of software systems. Nutrition ratio ratio management includes four user requests, namely, add, modify, delete and query. Business logic components need to define these four methods, the system controller components for the four interface programming, you can achieve the controller components and business logic components of the implementation of class separation.

(2) Business logic component implementation class. Implementation class is to achieve the interface of those methods of the method body, business logic component interface must be achieved through the implementation of specific business functions. In order for the business logic component to accept the Spring container into the DAO component, it is necessary to provide the corresponding setter method for the business logic component. The business logic class implements the operation of the database through the data persistence layer. Therefore, the business logic method needs to capture the data persistence layer exception and throw the user custom exception, so as to provide the operator with richer message.

(3) Configure business logic components. Including three configurations, one is to deploy the business logic component implementation class in the Spring container, and increase control for the business logic method; the second is to configure the business logic components rely on the DAO components; third, the business logic components to increase the transaction control, Spring for the 
BeanNameAutoProxyCreator post processor to the incoming transaction interceptor, the target bean generates the transaction proxy, so first define the BeanNameAutoProxyCreator post processor, and then configure the transaction manager bean and configure the transaction interceptor bean.

\section{Persistence Layer Implementation}

Persistence layer implementation is based on the data structure, the persistence of class design and mapping file design. Need to achieve all the criteria for the persistence layer, this article "lung capacity" as a standard, for example, to describe the persistence layer implementation.

(1) Data structure design. The data structure is organized by data elements based on some logical connection. The logical relationship between the data elements is called the logical structure of the data. The data must be stored in the computer, and the data storage structure is the implementation of the data structure. A logical data structure can have a variety of storage structures, and various storage structures affect the efficiency of data processing. The experience of many large systems shows that the difficulty of system implementation and the quality of system construction are heavily dependent on whether or not the optimal data structure is selected. In the software system, the data is usually stored in the database, this paper is based on SqlServer database management system design data structure to "lung capacity" standard logic as shown in Table 2.

Table 2. Logic structure of "VitalCapacity" standards

\begin{tabular}{ccccl}
\hline No & Name & Fields Type & Fields Width & \multicolumn{1}{c}{ Notes and Instructions } \\
\hline 1 & ID & Int & 4 & Primary key \\
2 & Grade & Varchar & 10 & Test grade \\
3 & Scores & Smallint & 2 & Individual Scores \\
4 & SchoolBoyA & Smallint & 2 & Schoolboy of freshman and sophomore \\
5 & SchoolGirlA & Smallint & 2 & Schoolgirl of freshman and sophomore \\
6 & SchoolBoyB & Smallint & 2 & Schoolboy junior and senior \\
7 & SchoolGiilB & Smallint & 2 & Schoolgirl junior and senior \\
8 & Remarks & Text & 8 & Remarks and instructions \\
\hline
\end{tabular}

(2) Data persistence design. For Hibernate PO, usually a persistent class is mapped to a data table, and the persistence class attribute corresponds to the data table column, so the system entity table contains a number of columns, it means that the entity corresponding to the persistence how many attributes are included in a class. PO is an object that incorporates Hibernate into its Entity Map, which represents a Hibernate entity that corresponds to a record in the database. The change in PO will be reflected in the actual database when the transaction is committed [9-10]. Persistent objects have the following characteristics: associated with the session instance; in the database associated with the record, there is a corresponding ID, in the memory of the object, the cache also. Hibernate is taken from the PO instance of the value, so even if the Session closed, the same can get / set, can be cross-session state management. "Vital capacity" table PO implementation code is as follows: 


\begin{tabular}{|c|c|}
\hline $\begin{array}{l}\text { public class VitalCapacity implements Serializable } \\
\text { \{ private Integer ID; } \\
\text { private String grade; } \\
\text { private Integer scores; } \\
\text { private Integer schoolBoyA; } \\
\text { private Integer schoolGirlA; } \\
\text { private Integer schoolBoyB; } \\
\text { private Integer schoolGirlB; } \\
\text { private String remarks; } \\
\text { private Set }<\text { Item }>\text { types = new HashSet }<\text { Item }>(\text { ); } \\
\text { public Set }<\text { Item }>\text { getTypes }() \\
\quad \text { \{ return types }\} \\
\text { public void setTypes (Set }<\text { Item }>\text { types }()\end{array}$ & $\begin{array}{l}\text { \{ this.types = types; }\} \\
\text { public void setID (Integer Id) } \\
\quad\{\text { this.Id = Id; }\} \\
\text { public Integer getID() } \\
\quad \text { \{ return Id; }\} \\
\text { public void setGrade (String Grade) } \\
\quad \text { this.Grade = Grade; } \\
\text { public String getGrade() } \\
\quad \text { \{ return Grade; }\} \\
\quad \text { // space limitations, } \\
\quad \text { //Other getter and setter methods } \\
\text { omitted } \\
\text { \} }\end{array}$ \\
\hline
\end{tabular}

(3) Mapping File. Mapping file is responsible for mapping the persistence class to the database table and mapping the attributes of the persistent class to the data column. Through this mapping, allowing the program to object-oriented way to operate Hibernate PO, and Hibernate is responsible for this operation into the underlying JDBC database access. The mapping file code corresponding to the above PO implementation is as follows:

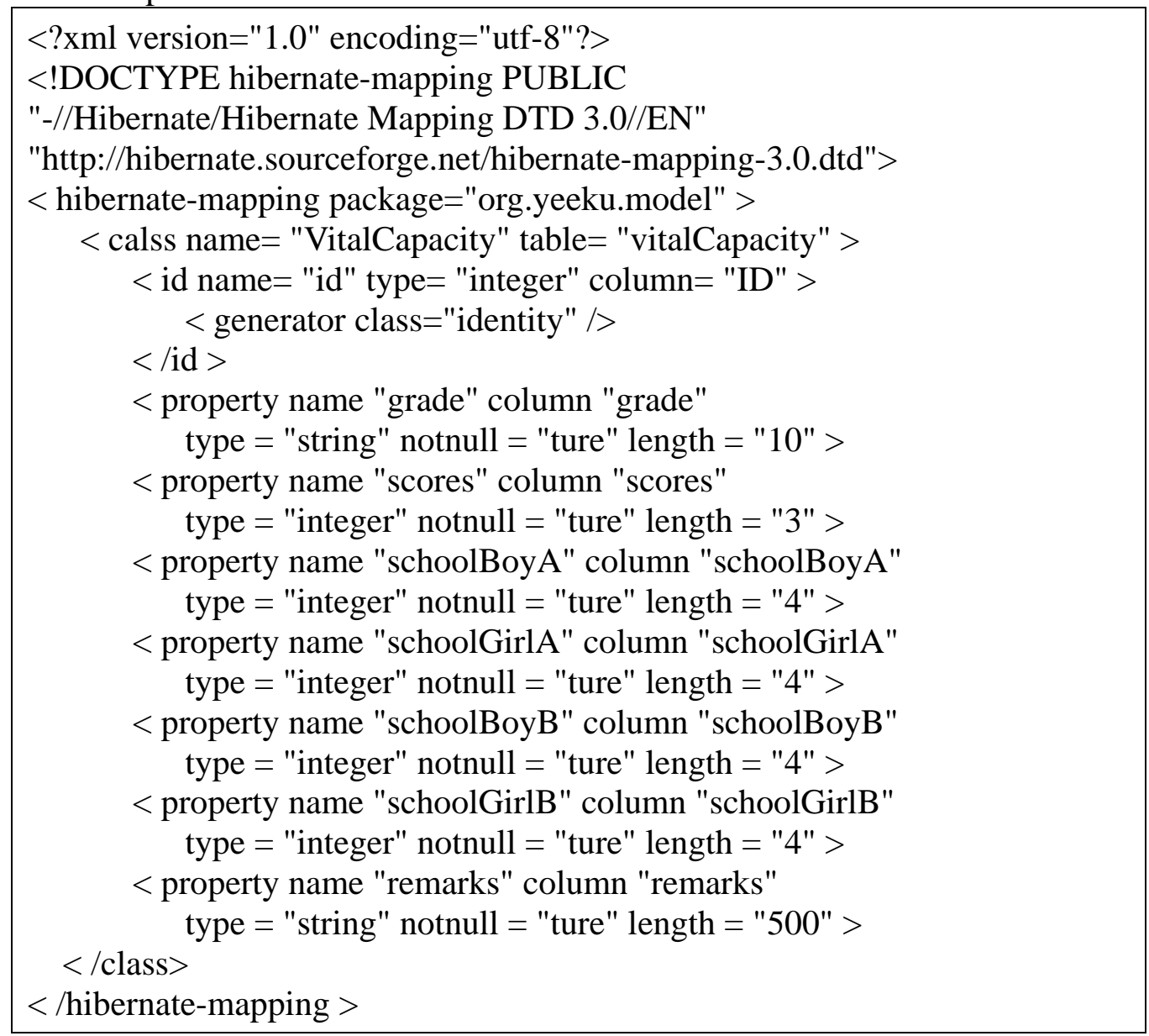

From the above mapping file is the PO entity (ResourceType) mapping to the data table (resourceType). The root element of the mapping file is class, and each class element is used to map a persistent class. The table attribute specified in the class element is the data table that indicates the persistent class map. If the table attribute is not specified, the name of the data table is the same as the name of the persistence class. Class element contains a number of property attributes, each property attribute mapping a persistent attribute, that is, to complete a property and a data field between the mapping. 


\section{Conclusion}

"Standard" uses individual evaluation criteria, set up special evaluation project for body shape, body function, physical fitness and athletic ability, can help students find their own shortcomings or individual differences, and through the evaluation to promote students to actively participate in physical exercise, through exercise to improve physical health, promote the overall development of body, become the high-quality builders with right sports awareness and healthy lifestyle, make school sports play its required role. This paper is designed to give full play to the advantages of SSH2 framework, Struts2 as controller, the view and business logic separated; Spring is responsible for bean management and transaction processing, integration Struts and Hibernate; Hibernate is responsible for interacting with the database. The research results of this paper solve the key technical problems in system development and have important guiding significance to improve the efficiency and quality of software development.

\section{References}

[1] J. Z. Guo, X. B. Huang, Z. Y. Hu, "Demonstration and Analysis on the Implementing Achievements of National Student Physical Health Standard in Higher Vocational Schools," Journal of Shaoguan University, vol. 38, no. 2, pp. 32-35, Feb. 2017.

[2] Baidu Knows. (2017, Apr). The implementation significance of national students' physique health standard. CN. [Online]. Available: https://zhidao.baidu.com/question/1110350633162843739.html.

[3] M. Luo, H. Wang, Z. C. Chen, "Development process research on National student's physical fitness and health standards," Sport Science and Technology, vol. 39, no. 1, pp. 100-101, Jan. 2017.

[4] C. W. Pu, C. F. Yang, Y. L. Bai, "Construction of Students' Conditioning Based on National Students' Physical Health Standard," Bulletin of Sport Science \& Technology, vol. 25, no. 4, pp. 1-2, Apr. 2017.

[5] Baidu library. (2017, Apr). National Undergraduate Physical Fitness Standard. CN. [Online]. Available: https://wenku.baidu.com/view/022614ecec3a87c24028c490.html.

[6] X. D. Liang, "Analysis of the influence of physical fitness standard test on physical exercise behavior of college students," Journal of Jinan Vocational College, vol. 18, no. 1, pp. 82-84, Jan. 2016.

[7] B. Chen, "Application of software configuration management in software development platform," Information \& Communications, vol. 28, no. 12, pp. 128 -128, Dec. 2014.

[8] L. J. Deng, Y. F. Zhang, J. M. Li, D. X. Dong, "Technology and application of SSI integration framework," Journal of Zhengzhou University of Light Industry(Natural Science Edition), vol. 30, no. 1, pp. 46-49, Jan. 2015.

[9] W. J. Tang, L. N. Chen, "Performance Optimization Scheme Research based on the Hibernate Lasting Layer," Intelligent Computer and Applications, vol. 2, no. 1, pp. 56-58, Jan. 2012.

[10] L. Guo, "Analysis of the Hibernate framework ORM common data association," Information system engineering, vol. 26, no. 4, pp. 134, Apr. 2013. 